\title{
Identities on generalized Fibonacci and Lucas numbers
}

\author{
K. M. Nagaraja ${ }^{1}$ and P. Dhanya ${ }^{2}$ \\ ${ }^{1}$ Department of Mathematics, J.S.S. Academy of Technical Education \\ Uttarahalli-Kengeri Main Road, Bengaluru-60, Karnataka, India \\ e-mail: nagkmn@gmail.com \\ ${ }^{2}$ Department of Mathematics, J.S.S. Academy of Technical Education \\ Uttarahalli-Kengeri Main Road, Bengaluru-60, Karnataka, India \\ e-mail: dhanyap.kglegmail.com
}

\begin{abstract}
In this article, the concepts of Fibonacci, Tribonacci, Lucas and Tetranacci numbers are generalized as continued sum. The generalized Fibonacci identity is proved by using induction and the binomial theorem. Further, it is proved that the generalized Fibonacci and Lucas sequences are logarithmically convex (concave) and some special identities are obtained.
\end{abstract} Keywords: Sequence, Fibonacci number, Lucas number, Tribonacci number, Golden ratio. 2010 Mathematics Subject Classification: 11B39.

\section{Introduction}

A sequence is an arrangement of objects or a set of numbers in a particular order. Let $\left\{u_{n}\right\}$ be any sequence of terms $u_{0}, u_{1}, u_{2}, \ldots$ in which the $n$-th and $(n+1)$-st terms of the sequence are respectively denoted by $u_{n}$ and $u_{n+1}$. If $u_{n+1}>(<) u_{n}$, then sequence $\left\{u_{n}\right\}$ is monotonically increasing (decreasing) $[12,16]$.

Fibonacci sequence: The sequence of numbers of the form $\{0,1,1,2,3,5,8, \ldots\}$ is called the Fibonacci sequence. The numbers $0,1,1,2, \ldots$ are called Fibonacci numbers. In 1202, Fibonacci used this concept to study the growth of rabbit population and some interesting results are found in $[3,5-7,11]$. The general term of the Fibonacci sequence is denoted by $F_{n}$ and defined as follows. 
Definition 1.1. For all positive integers $n$,

$$
F_{n}= \begin{cases}0, & \text { if } n=0 \\ 1, & \text { if } n=1 \\ F_{n-1}+F_{n-2}, & \text { if } n \geq 2\end{cases}
$$

with $F_{0}=0, F_{1}=1, F_{2}=1, F_{3}=2, \ldots$.

In general, any number is obtained by adding previous two terms.

Tribonacci sequence: A Tribonacci sequence is denoted by $\left\{T_{n}\right\}$, which is the generalized Fibonacci sequence. Here $T_{0}=0, T_{1}=1, T_{2}=1$, the terms $T_{3}$ onwards are obtained by adding previous three terms. That means, $T_{n}=T_{n-1}+T_{n-2}+T_{n-3}$ for $n \geq 3$. The terms of the sequence are $\{0,1,1,2,4,7, \ldots\}$. The results and identities on Tribonacci numbers are discussed in $[1,4,6,7]$.

Lucas sequence: The Lucas sequence, named after the mathematician François Édouard Anatole Lucas (1842-1891), is closely related to the Fibonacci sequence. The Lucas sequence has the same recursive relationship as the Fibonacci sequence, where each term is the sum of the previous two terms, but with different initial values. The sequence of the form $\{2,1,3,4,7,11,18,29$, $47, \ldots\}$ is called Lucas sequence. The numbers $2,1,3,4, \ldots$ are called Lucas numbers and some interesting results are found in $[7,11,15]$. The general term of the Lucas sequence is denoted by $L_{n}$ and defined as follows.

Definition 1.2. For all positive integers $n$,

$$
L_{n}= \begin{cases}2, & \text { if } n=0 \\ 1, & \text { if } n=1 \\ L_{n-1}+L_{n-2}, & \text { if } n \geq 2\end{cases}
$$

with $L_{0}=2, L_{1}=1, L_{2}=3, L_{3}=4, \ldots$.

In general, any number is obtained by adding previous two numbers with the first two numbers are 2 and 1 . The following are the identities involving Fibonacci and Lucas numbers $[8,9]$.

$$
\begin{gathered}
(-1)^{n+1}=F_{n-1} F_{n+1}-F_{n}^{2} \\
F_{m+n-1}=F_{m} F_{n}+F_{m-1} F_{n-1} \\
F_{m+n}=F_{m} F_{n+1}+F_{m-1} F_{n}=L_{n} F_{n}-(-1)^{n} F_{n-m} \\
L_{n+3}=F_{n}+F_{n+1}+F_{n+2}+F_{n+3} \\
L_{n}^{2}=5 F_{n}^{2}+4(-1)^{n} \\
F_{n}=\frac{L_{n-1}+L_{n+1}=\frac{L_{n}+F_{n}}{2}}{5} \\
F_{n+m}=\frac{L_{m} F_{n}+L_{n} F_{m}}{2} \\
L_{n+m}=L_{m+1} F_{n}+L_{m} F_{n-1}=\frac{L_{m} L_{n}+5 F_{n} F_{m}}{2}=(-1)^{m} L_{n-m}+5 L_{n} F_{m}
\end{gathered}
$$


D'Ocagne's identity:

$$
\begin{gathered}
F_{m} F_{n+1}-F_{n} F_{m+1}=(-1)^{n} F_{m-n} \\
F_{-n}=(-1)^{n+1} F_{n}, \quad \text { for } \quad n=1, F_{-1}=1
\end{gathered}
$$

In [2], Zvonko Cerin studied on factors of sums of consecutive Fibonacci and Lucas numbers. The author discovered that the sums $\sum_{j=0}^{4 i+3} F_{k+j}$ have the Fibonacci number $F_{2 i+2}$ as a common factor, the alternating sums of 20 and 22 consecutive Fibonacci numbers are all respectively divisible by $F_{10}$ and $L_{11}$. Also, obtained some interesting results on sums of consecutive products, and squares of consecutive numbers. Below are given few identities involving Fibonacci and Lucas numbers [2].

$$
\begin{array}{cc}
\sum_{j=0}^{4 i+3} F_{k+j}=F_{2 i+2} L_{k+2 i+3} \quad \text { and } & \sum_{j=0}^{4 i+3}(-1)^{j} F_{k+j}=F_{2 i+2} L_{k+2 i} \\
\sum_{j=0}^{4 i+1} F_{k+j}=L_{2 i+1} F_{k+2 i+2} & \text { and } \quad \sum_{j=0}^{4 i+1}(-1)^{j} F_{k+j}=L_{2 i+1} F_{k+2 i-1} \\
\sum_{j=0}^{4 i} F_{k+j}=F_{2 i} L_{k+2 i}+L_{2 i+1} F_{k+2 i} & \text { and } \quad \sum_{j=0}^{4 i}(-1)^{j} F_{k+j}=F_{k+2 i} L_{2 i+1}-L_{k+2 i} F_{2 i}
\end{array}
$$

and for other identities interested readers may refer [2].

Golden ratio: Let $u_{n}$ and $u_{n+1}$ be any two consecutive terms of monotonically increasing sequence. If

$$
\lim _{n \rightarrow \infty} \frac{u_{n+1}}{u_{n}}=1.6180339887 \ldots,
$$

then, the ratio $\frac{u_{n+1}}{u_{n}}$ is called a Golden ratio and is denoted by the Greek alphabet $\varphi$. The value of $\varphi=\frac{1+\sqrt{5}}{2}=1.6180339887 \ldots$ and $1-\varphi=\phi=\frac{1-\sqrt{5}}{2}=-0.6180339887 \ldots$ Both $\varphi$ and $\phi$ are the solutions of the quadratic equation $x^{2}-x-1=0$, a good number of results on Golden ratio are found in $[3-5,11]$.

Definition 1.3 ([16]). A sequence $\left\{u_{n}\right\}$ is said to be log-convex if $\left(u_{n+1}\right)^{2}<u_{n} u_{n+2}$ and is log-concave if $\left(u_{n+1}\right)^{2}>u_{n} u_{n+2}$ for all $n$, some interesting results on convexities are found in $[13,14]$.

\section{Generalization of Fibonacci numbers}

From the motivation of the above literature survey, consider a set of new sequences of the form:

$$
\begin{aligned}
D_{j}^{0} & =\{0,1,1,2,3, \ldots\}=F_{j}, \text { for all } j=0,1,2, \ldots \\
D_{j}^{1} & =\{0+1=\mathbf{1}, 1+1=\mathbf{2}, 1+2=\mathbf{3}, 2+3=\mathbf{5}, \ldots\} \\
& =F_{j}+F_{j+1}, \text { for all } j=0,1,2, \ldots \\
D_{j}^{2} & =\{0+1+1=, \mathbf{2}, 1+1+2=\mathbf{4}, 1+2+3=\mathbf{6}, \ldots\} \\
& =F_{j}+F_{j+1}+F_{j+2}, \text { for all } j=0,1,2, \ldots \\
D_{j}^{3} & =\{0+1+1+2=\mathbf{4}, 1+1+2+3=\mathbf{7}, 1+2+3+5=\mathbf{1 1}, \ldots\} \\
& =F_{j}+F_{j+1}+F_{j+2}+F_{j+3} \\
& =L_{j+3}, \text { for all } j=0,1,2, \ldots \quad \text { and so on. }
\end{aligned}
$$


The family of sequences $\left\{D_{j}^{k}\right\}, k=0,1,2, \ldots$ is generalized as follows.

Definition 2.1. For any two positive integers $j$ and $k$, the generalized Fibonacci sequence $\left\{D_{j}^{k}\right\}$ is defined as:

$$
D_{j}^{k}=F_{j}+F_{j+1}+F_{j+2}+\ldots+F_{j+k-1}+F_{j+k}=\sum_{i=j}^{j+k} F_{i}
$$

where $j=0,1,2, \ldots$ and $k=0,1,2, \ldots$

Obviously the sequence $\left\{D_{j}^{k}\right\}$ is monotonically increasing sequence and the following identities are obtained.

$$
\begin{aligned}
& D_{j+2}^{k}=D_{j}^{k}+D_{j+1}^{k}, \quad \text { for all } j, k=0,1,2,3, \ldots \\
& D_{j}^{k+1}-D_{j}^{k}=D_{j+k+1}^{0}, \quad \text { for all } j, k=0,1,2,3, \ldots \\
& D_{j}^{k+1}-D_{j+1}^{k}=F_{j}, \quad \text { for all } j, k=0,1,2,3, \ldots \\
& D_{j}^{2}=2 D_{j}^{1}, \quad \text { for all } j=0,1,2,3, \ldots
\end{aligned}
$$

The first 10 numbers of the sequence $\left\{D_{j}^{k}\right\}$ are represented in the form of the table as below.

\begin{tabular}{|r|r|r|r|r|r|r|r|r|l|}
\hline$C$ & $C_{0}$ & $C_{1}$ & $C_{2}$ & $C_{3}$ & $C_{4}$ & $C_{5}$ & $C_{6}$ & $C_{7}$ & $\ldots$ \\
\hline$j$ & $D_{j}^{0}$ & $D_{j}^{1}$ & $D_{j}^{2}$ & $D_{j}^{3}$ & $D_{j}^{4}$ & $D_{j}^{5}$ & $D_{j}^{6}$ & $D_{j}^{7}$ & $\ldots$ \\
\hline 0 & 0 & 1 & 2 & 4 & 7 & 12 & 20 & 33 & $\ldots$ \\
1 & 1 & 2 & 4 & 7 & 12 & 20 & 33 & 54 & $\ldots$ \\
2 & 1 & 3 & 6 & 11 & 19 & 32 & 53 & 87 & $\ldots$ \\
3 & 2 & 5 & 10 & 18 & 31 & 52 & 86 & 141 & $\ldots$ \\
4 & 3 & 8 & 16 & 29 & 50 & 84 & 139 & 228 & $\ldots$ \\
5 & 5 & 13 & 26 & 47 & 81 & 136 & 225 & 369 & $\ldots$ \\
6 & 8 & 21 & 42 & 76 & 131 & 220 & 364 & 597 & $\ldots$ \\
7 & 13 & 34 & 68 & 123 & 212 & 356 & 589 & 966 & $\ldots$ \\
8 & 21 & 55 & 110 & 199 & 343 & 576 & 953 & 1563 & $\ldots$ \\
9 & 34 & 89 & 178 & 322 & 555 & 932 & 1542 & 2529 & $\ldots$ \\
$\vdots$ & $\vdots$ & $\vdots$ & $\vdots$ & $\vdots$ & $\vdots$ & $\vdots$ & $\vdots$ & $\vdots$ & $\ddots$ \\
\hline
\end{tabular}

Table 2.1. The first 10 numbers of the sequence $\left\{D_{j}^{k}\right\}$

Note: The results studied by Zvonko Cerin [2] correspond to the columns $C_{3}, C_{7}, C_{11}, C_{15}$, $\ldots, C_{4 i+3}$ where $i=0,1,2,3, \ldots$.

The objective of this article is to develop some identities involving the generalized Fibonacci sequence and to prove the sequence is log-convex (concave) for the set of even (odd) numbers.

\section{Main results on the generalized Fibonacci sequence}

Theorem 3.1. If $\left\{D_{j}^{k}\right\}$ is the generalized Fibonacci sequence, then $\lim _{j \rightarrow \infty} \frac{D_{j+1}^{k}}{D_{j}^{k}}$ converges to the Golden ratio. 
Proof. Consider the ratio of two consecutive terms of the generalized Fibonacci sequence and using the identity (2.2) gives

$$
\frac{D_{j+1}^{k}}{D_{j}^{k}}=\frac{D_{j-1}^{k}+D_{j}^{k}}{D_{j}^{k}}=1+\frac{D_{j-1}^{k}}{D_{j}^{k}} .
$$

Define a quantity $x=\lim _{j \rightarrow \infty} \frac{D_{j+1}^{k}}{D_{j}^{k}}$, and its reciprocal $\frac{1}{x}=\lim _{j \rightarrow \infty} \frac{D_{j}^{k}}{D_{j+1}^{k}}$.

Also, the limit can be written as $\lim _{j \rightarrow \infty} \frac{D_{j-1}^{k}}{D_{j}^{k}}$, therefore $\frac{1}{x}=\lim _{j \rightarrow \infty} \frac{D_{j}^{k}}{D_{j+1}^{k}}=\lim _{j \rightarrow \infty} \frac{D_{j-1}^{k}}{D_{j}^{k}}$.

As $j$ tends to $\infty$, equation (3.1) gives,

$$
\lim _{j \rightarrow \infty} \frac{D_{j+1}^{k}}{D_{j}^{k}}=\lim _{j \rightarrow \infty}\left[1+\frac{D_{j-1}^{k}}{D_{j}^{k}}\right]=1+\lim _{j \rightarrow \infty}\left[\frac{D_{j-1}^{k}}{D_{j}^{k}}\right]
$$

which is equivalently, $x=1+\frac{1}{x}$ or $x^{2}=x+1$ or $x^{2}-x-1=0$.

The roots of above equation are $\frac{1+\sqrt{5}}{2}$ and $\frac{1-\sqrt{5}}{2}$, i.e., $x=\lim _{j \rightarrow \infty} \frac{D_{j+1}^{k}}{D_{j}^{k}}=\varphi=\frac{1+\sqrt{5}}{2}$, which is the value of the Golden ratio.

Lemma 3.1. Let $\varphi$ be a Golden ratio and $\phi=1-\varphi$, then $\varphi^{n}+\phi^{n}=1-\sum_{k=1}^{n-1}{ }^{n} c_{k}(\varphi)^{n-k} \phi^{k}$.

Proof. The binomial theorem states that for all integer values of $n$,

$$
(a+b)^{n}=\sum_{k=0}^{n}{ }^{n} c_{k} a^{n-k} b^{k}=a^{n}+{ }^{n} c_{1} a^{n-1} b+{ }^{n} c_{2} a^{n-2} b^{2}+{ }^{n} c_{3} a^{n-3} b^{3}+\ldots+b^{n} .
$$

Consider $a=\left(\frac{1+\sqrt{5}}{2}\right)=\varphi$ and $b=\left(\frac{1-\sqrt{5}}{2}\right)=\phi$, then $a^{n}=\left(\frac{1+\sqrt{5}}{2}\right)^{n}=\varphi^{n}$ and $b^{n}=\left(\frac{1-\sqrt{5}}{2}\right)^{n}=\phi^{n}$.

By binomial theorem,

$$
\varphi^{n}=\frac{1}{2^{n}}\left[1+{ }^{n} c_{1} \sqrt{5}+{ }^{n} c_{2} 5+{ }^{n} c_{3} 5 \sqrt{5}+\ldots\right]
$$

and

$$
\phi^{n}=\frac{1}{2^{n}}\left[1-{ }^{n} c_{1} \sqrt{5}+{ }^{n} c_{2} 5-{ }^{n} c_{3} 5 \sqrt{5}+\ldots\right] .
$$

Adding gives

$$
\varphi^{n}+\phi^{n}=\frac{1}{2^{n-1}}\left[1+{ }^{n} c_{2}(\sqrt{5})^{2}+{ }^{n} c_{4}(\sqrt{5})^{4}+{ }^{n} c_{6}(\sqrt{5})^{6}+\ldots\right]=\frac{1}{2^{n-1}} \sum_{r=0}^{n}{ }^{n} c_{2 r}(\sqrt{5})^{2 r} \text {. }
$$

Since the binomial theorem holds for all integer values of $n$, then

$$
\varphi^{n+1}+\phi^{n+1}=\frac{1}{2^{n}}\left[\sum_{r=0}^{n+1}{ }^{n+1} c_{2 r}(\sqrt{5})^{2 r}\right] .
$$

For $j=0,1,2, \ldots$ and $k=0,1,2, \ldots$, the main identity of this article is stated as below.

$$
\Delta_{j}^{k}=\left(D_{j+1}^{k}\right)^{2}-D_{j}^{k} D_{j+2}^{k}=(-1)^{j}\left[(-1)^{k}+\left(\frac{1-\sqrt{5}}{2}\right)^{k+1}+\left(\frac{1+\sqrt{5}}{2}\right)^{k+1}-1\right]
$$


By using Lemma 3.1,

$$
\Delta_{j}^{k}=\left(D_{j+1}^{k}\right)^{2}-D_{j}^{k} D_{j+2}^{k}=(-1)^{j}\left[(-1)^{k}-1+\frac{1}{2^{k}} \sum_{r=0}^{k+1}{ }^{k+1} c_{2 r}(\sqrt{5})^{2 r}\right]
$$

Theorem 3.2. Let $\left\{D_{j}^{k}\right\}$ be the generalized Fibonacci sequence, then

$$
\left(D_{j+1}^{k}\right)^{2}-D_{j}^{k} D_{j+2}^{k}=(-1)^{j}\left[(-1)^{k}+\left(\frac{1-\sqrt{5}}{2}\right)^{k+1}+\left(\frac{1+\sqrt{5}}{2}\right)^{k+1}-1\right]
$$

is log-convex for $j=0,2,4,6, \ldots$ and log-concave for $j=1,3,5, \ldots$.

Proof. The theorem is proved by induction and the binomial theorem.

Case 1. Let $k$ be fixed and $j$ vary.

$$
\begin{aligned}
& \text { Put } k=0, j=0,\left(D_{1}^{0}\right)^{2}-D_{0}^{0} D_{2}^{0}=(-1)^{0}\left[(-1)^{0}+\frac{1-\sqrt{5}}{2}+\frac{1+\sqrt{5}}{2}-1\right]=1 . \\
& \text { Put } k=0, j=1,\left(D_{2}^{0}\right)^{2}-D_{1}^{0} D_{3}^{0}=(-1)^{1}\left[(-1)^{1}+\frac{1-\sqrt{5}}{2}+\frac{1+\sqrt{5}}{2}-1\right]=1 .
\end{aligned}
$$

So, it holds for $k=0, j=0$ and 1 .

Assume that the identity (3.2) holds true for fixed $k$ and $j=m$, that is,

$$
\left(D_{m+1}^{k}\right)^{2}-D_{m}^{k} D_{m+2}^{k}=(-1)^{m}\left[(-1)^{k}+\left(\frac{1-\sqrt{5}}{2}\right)^{k+1}+\left(\frac{1+\sqrt{5}}{2}\right)^{k+1}-1\right] \text {. }
$$

To prove the identity holds true for fixed $k$ and $j=m+1$, by using the identity (2.2) gives

$$
\left(D_{m+2}^{k}\right)^{2}-D_{m+1}^{k} D_{m+3}^{k}=\left(D_{m+1}^{k}+D_{m}^{k}\right)^{2}-D_{m+1}^{k}\left(D_{m+1}^{k}+D_{m+2}^{k}\right) .
$$

By simplification of the right-hand side, we have

$$
\begin{aligned}
& =\left(D_{m+1}^{k}\right)^{2}+\left(D_{m}^{k}\right)^{2}+2 D_{m+1}^{k} D_{m}^{k}-\left(D_{m+1}^{k}\right)^{2}-D_{m+1}^{k} D_{m+2}^{k} \\
& =\left(D_{m}^{k}\right)^{2}+D_{m+1}^{k} D_{m}^{k}-D_{m+1}^{k} D_{m+2}^{k}+D_{m+1}^{k} D_{m}^{k} \\
& =D_{m}^{k}\left(D_{m}^{k}+D_{m+1}^{k}\right)-D_{m+1}^{k}\left(D_{m+2}^{k}-D_{m}^{k}\right) \\
& =-\left[\left(D_{m+1}^{k}\right)^{2}-D_{m}^{k} D_{m+2}^{k}\right] .
\end{aligned}
$$

From equation (3.2)

$$
\begin{aligned}
& =-(-1)^{j}\left[(-1)^{k}+\left(\frac{1-\sqrt{5}}{2}\right)^{k+1}+\left(\frac{1+\sqrt{5}}{2}\right)^{k+1}-1\right] \\
& =(-1)^{j+1}\left[(-1)^{k}+\left(\frac{1-\sqrt{5}}{2}\right)^{k+1}+\left(\frac{1+\sqrt{5}}{2}\right)^{k+1}-1\right] .
\end{aligned}
$$

This proves that the identity (3.2) holds true for all positive integer $j$ by induction. 
Case 2. Let $j$ be fixed and $k$ vary.

Put $j=0, k=0,\left(D_{1}^{0}\right)^{2}-D_{0}^{0} D_{2}^{0}=(-1)^{0}\left[(-1)^{0}+\frac{1-\sqrt{5}}{2}+\frac{1+\sqrt{5}}{2}-1\right]=1$.

Put $j=0, k=1,\left(D_{1}^{1}\right)^{2}-D_{0}^{1} D_{2}^{1}=(-1)^{0}\left[(-1)^{1}+\left(\frac{1-\sqrt{5}}{2}\right)^{2}+\left(\frac{1+\sqrt{5}}{2}\right)^{2}-1\right]=1$.

So, it holds for $j=0, k=0$ and 1 .

Assume that the identity (3.2) holds true for fixed $j$ and $k=m$, that is

$$
\begin{gathered}
\left(D_{j+1}^{m}\right)^{2}-D_{j}^{m} D_{j+2}^{m}=(-1)^{j}\left[(-1)^{m}+\left(\frac{1-\sqrt{5}}{2}\right)^{m+1}+\left(\frac{1+\sqrt{5}}{2}\right)^{m+1}-1\right] \\
=(-1)^{j}\left[(-1)^{m}-1+\frac{1}{2^{m}} \sum_{r=0}^{m+1}{ }^{m+1} c_{2 r}(\sqrt{5})^{2 r}\right] .
\end{gathered}
$$

To prove that the identity (3.2) holds true for fixed $j$ and $k=m+1$.

Consider the right-hand side of equation (3.3) when $k=m+1$,

By Lemma 3.1

$$
\begin{aligned}
& =(-1)^{j}\left[(-1)^{m+1}+\left(\frac{1-\sqrt{5}}{2}\right)^{m+2}+\left(\frac{1+\sqrt{5}}{2}\right)^{m+2}-1\right] \\
& =(-1)^{j}\left[(-1)^{m+1}-1+\frac{1}{2^{m+1}} \sum_{r=0}^{m+2}{ }^{m+2} c_{2 r}(\sqrt{5})^{2 r}\right] .
\end{aligned}
$$

$$
\begin{aligned}
& =(-1)^{j}\left[(-1)^{m+1}-1+\varphi^{k+2}+\phi^{k+2}\right] \\
& =\left(D_{j+1}^{m+1}\right)^{2}-D_{j}^{m+1} D_{j+2}^{m+1} .
\end{aligned}
$$

This proves that the identity (3.2) holds for all positive integers of $k$ by induction.

Theorem 3.2 is illustrated for the first 10 values of $j=0,1,2, \ldots, k=0,1,2, \ldots$ and the values of $\left(D_{j+1}^{k}\right)^{2}-D_{j}^{k} D_{j+2}^{k}$ are represented in the form of the table below.

\begin{tabular}{|r|r|r|r|r|r|r|r|r|r|r|l|}
\hline$C$ & $C_{0}$ & $C_{1}$ & $C_{2}$ & $C_{3}$ & $C_{4}$ & $C_{5}$ & $C_{6}$ & $C_{7}$ & $C_{8}$ & $C_{9}$ & $\ldots$ \\
\hline$j$ & $D_{j}^{0}$ & $D_{j}^{1}$ & $D_{j}^{2}$ & $D_{j}^{3}$ & $D_{j}^{4}$ & $\Delta_{j}^{0}$ & $\Delta_{j}^{1}$ & $\Delta_{j}^{2}$ & $\Delta_{j}^{3}$ & $\Delta_{j}^{4}$ & $\ldots$ \\
\hline 0 & 0 & 1 & 2 & 4 & 7 & - & - & - & - & - & $\ldots$ \\
1 & 1 & 2 & 4 & 7 & 12 & -1 & -1 & -4 & -5 & -11 & $\ldots$ \\
2 & 1 & 3 & 6 & 11 & 19 & 1 & 1 & 4 & 5 & 11 & $\ldots$ \\
3 & 2 & 5 & 10 & 18 & 31 & -1 & -1 & -4 & -5 & -11 & $\ldots$ \\
4 & 3 & 8 & 16 & 29 & 50 & 1 & 1 & 4 & 5 & 11 & $\ldots$ \\
5 & 5 & 13 & 26 & 47 & 81 & -1 & -1 & -4 & -5 & -11 & $\ldots$ \\
6 & 8 & 21 & 42 & 76 & 131 & 1 & 1 & 4 & 5 & 11 & $\ldots$ \\
7 & 13 & 34 & 68 & 123 & 212 & -1 & -1 & -4 & -5 & -11 & $\ldots$ \\
8 & 21 & 55 & 110 & 199 & 343 & 1 & 1 & 4 & 5 & 11 & $\ldots$ \\
9 & 34 & 89 & 178 & 322 & 555 & -1 & -1 & -4 & -5 & -11 & $\ldots$ \\
$\vdots$ & $\vdots$ & $\vdots$ & $\vdots$ & $\vdots$ & $\vdots$ & $\vdots$ & $\vdots$ & $\vdots$ & $\vdots$ & $\vdots$ & $\ddots$ \\
\hline
\end{tabular}

Table 3.1. The first 10 numbers of the sequence represents $\left\{\left(D_{j+1}^{k}\right)^{2}-D_{j}^{k} D_{j+2}^{k}\right\}$ 
It is observed that from the above table the values of $\Delta_{j}^{k}$ are negative (log-convex) for $j=1,3,5,7, \ldots$ and positive (log-concave) for $j=2,4,6,8, \ldots$

Remark 3.1. If $\left\{L_{n}\right\}$ is the Lucas sequence, then

1. $L_{n+3}$ is log-convex if $n$ is even.

2. $L_{n+3}$ is log-concave if $n$ is odd.

Proof. Let $L_{n+3}$ be a Lucas number, then by using equation (1.4) in $L_{n+4}^{2}-L_{n+3} L_{n+5}$ equal to:

$=\left(F_{n+1}+F_{n+2}+F_{n+3}+F_{n+4}\right)^{2}-\left(F_{n}+F_{n+1}+F_{n+2}+F_{n+3}\right)\left(F_{n+2}+F_{n+3}+F_{n+4}+F_{n+5}\right)$

$=\left(2 F_{n+3}+F_{n+4}\right)^{2}-\left(2 F_{n+2}+F_{n+3}\right)\left(2 F_{n+4}+F_{n+5}\right)$

$=\left(4 F_{n+3}^{2}+F_{n+4}^{2}+4 F_{n+3} F_{n+4}-\right)-\left(4 F_{n+2} F_{n+4}+2 F_{n+3} F_{n+4}+2 F_{n+2} F_{n+5}+F_{n+3} F_{n+5}\right)$.

By using identity (1.1),

$=4(-1)^{n+4}+(-1)^{n+5}+2\left(F_{n+3} F_{n+4}-F_{n+2} F_{n+5}\right)$

$=4(-1)^{n}-(-1)^{n}+2\left(F_{n+4} F_{n+3}-F_{n+5} F_{n+2}\right)$.

By using identity (1.9),

$=3(-1)^{n}+2(-1)^{n}$

$=5(-1)^{n}$.

$\therefore$ If $n$ is even, then $L_{n+3}$ is log-convex, otherwise it is log-concave.

The above remark is illustrated by using system software for $1 \leq i \leq 37$.

\begin{tabular}{|r|r|c|c|}
\hline$n$ & $L_{n}$ & $\left(L_{n}\right)^{2}-L_{n-1} L_{n+1}$ & Result \\
\hline 0 & 2 & - & - \\
1 & 1 & -5 & convexity \\
2 & 3 & 5 & concavity \\
3 & 4 & -5 & convexity \\
4 & 7 & 5 & concavity \\
5 & 11 & -5 & convexity \\
6 & 18 & 5 & concavity \\
7 & 29 & -5 & convexity \\
8 & 47 & 5 & concavity \\
9 & 76 & -5 & convexity \\
$\vdots$ & $\vdots$ & $\vdots$ & $\vdots$ \\
\hline
\end{tabular}

Table 3.2. The first 10 Lucas numbers represent $\left\{\left(L_{n}\right)^{2}-L_{n-1} L_{n+1}\right\}$

Theorem 3.3. For all positive integers $j$ and $k, D_{j}^{k}=\frac{L_{j+1} F_{k+1}+F_{j+1} L_{k+1}-2 F_{j+1}}{2}$.

Proof. This identity is proved by induction. 


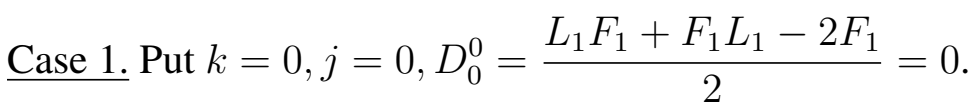

Put $k=0, j=1, D_{1}^{0}=\frac{L_{2} F_{1}+F_{2} L_{1}-2 F_{2}}{2}=1$.

So, it holds for $k=0, j=0$ and 1 . Assume that identity holds for fixed $k$ and $j=m$.

That is, $D_{m}^{k}=\frac{L_{m+1} F_{k+1}+F_{m+1} L_{k+1}-2 F_{m+1}}{2}$.

To prove the identity holds for fixed $k$ and $j=m+1$, by Definition 2.1 and by using the identity (1.7) gives,

On simplification,

$$
\begin{aligned}
D_{m+1}^{k} & =D_{m}^{k}+F_{m+k+1}-F_{m} \\
& =\frac{L_{m+1} F_{k+1}+F_{m+1} L_{k+1}-2 F_{m+1}}{2}+\frac{L_{m} F_{k+1}+F_{m} L_{k+1}}{2}-F_{m} \\
& =\frac{L_{m+1} F_{k+1}+F_{m+1} L_{k+1}-2 F_{m+1}+L_{m} F_{k+1}+F_{m} L_{k+1}}{2}-\frac{2 F_{m}}{2} .
\end{aligned}
$$

$$
D_{m+1}^{k}=\frac{L_{m+2} F_{k+1}+F_{m+2} L_{k+1}-2 F_{m+2}}{2}
$$

It holds for all positive integer $j$.

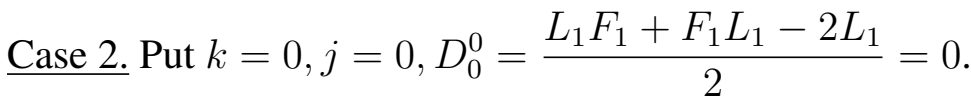

Put $k=1, j=0, D_{0}^{1}=\frac{L_{1} F_{2}+F_{1} L_{2}-2 F_{1}}{2}=1$.

So, it holds true for $j=0$ and $k=0$ and 1 .

Assume that it holds good for fixed $j$ and $k=m$,

i.e., $D_{j}^{m}=\frac{L_{j+1} F_{m+1}+F_{j+1} L_{m+1}-2 F_{j+1}}{2}$.

To prove the identity holds good for fixed $j$ and $k=m+1$, by Definition 2.1 and by using the identity (1.7) gives,

$$
\begin{aligned}
D_{j}^{m+1} & =D_{j}^{m}+F_{j+m+1} \\
& =\frac{L_{j+1} F_{m+1}+F_{j+1} L_{m+1}-2 F_{j+1}}{2}+\frac{L_{j+1} F_{m}+F_{j+1} L_{m}}{2} \\
& =\frac{L_{j+1} F_{m+1}+F_{j+1} L_{m+1}-2 F_{j+1}+L_{j+1} F_{m}+F_{j+1} L_{m}}{2} .
\end{aligned}
$$

On simplification,

$$
D_{j}^{m+1}=\frac{L_{j+1} F_{m+2}+F_{j+1} L_{m+2}-2 F_{j+1}}{2} .
$$

It holds true for positive integer $k$. Hence the theorem is proved. 
Remark 3.2. For particular values of $j, D_{j}^{k}=\frac{L_{j+1} F_{k+1}+F_{j+1} L_{k+1}-2 F_{j+1}}{2}$ satisfies the following identities:
1. $D_{0}^{k}=\frac{F_{k+1}+L_{k+1}-2}{2}$
2. $D_{1}^{k}=\frac{3 F_{k+1}+L_{k+1}-2}{2}$
3. $D_{3}^{k}=\frac{4 F_{k+1}+2 L_{k+1}-4}{2}$

Remark 3.3. Let $\left\{D_{j}^{k}\right\}$ be the generalized Fibonacci sequence, then for any positive integer a the following identity holds.

$$
D_{j}^{k}-D_{j-a}^{k} D_{j+a}^{k}=(-1)^{j+a}\left[(-1)^{k}+\left(\frac{1-\sqrt{5}}{2}\right)^{k+1}+\left(\frac{1+\sqrt{5}}{2}\right)^{k+1}-1\right]\left(D_{a}^{0}\right)^{2}
$$

\section{Generalization of Lucas numbers}

A set of new sequences is defined by using Lucas numbers. Consider a set of new sequences of the form.

$$
\begin{aligned}
E_{j}^{0} & =\{2,1,3,4,7, \ldots\}=L_{j} \text { for all } j=0,1,2, \ldots \\
E_{j}^{1} & =\{2+1=\mathbf{3}, 1+3=\mathbf{4}, 3+4=\mathbf{7}, 4+7=\mathbf{1 1}, 7+11=\mathbf{1 8}, \ldots\} \\
& =L_{j}+L_{j+1} \text { for all } j=0,1,2, \ldots \\
E_{j}^{2} & =\{2+1+3=\mathbf{6}, 1+3+4=\mathbf{8}, 3+4+7=\mathbf{1 4}, 4+7+11=\mathbf{2 2}, 7+11+18=\mathbf{3 6}, \ldots\} \\
& =L_{j}+L_{j+1}+L_{j+2} \text { for all } j=0,1,2, \ldots \text { and so on. }
\end{aligned}
$$

The family of sequences $\left\{E_{j}^{k}\right\}, k=0,1,2, \ldots$ is generalized as follows.

Definition 4.1. For two positive integers $j$ and $k$, the generalized Lucas sequence $\left\{E_{j}^{k}\right\}$ is defined as;

$$
E_{j}^{k}=L_{j}+L_{j+1}+L_{j+2}+\ldots+L_{j+k-1}+L_{j+k}=\sum_{i=j}^{j+k} L_{i}
$$

where $j=0,1,2, \ldots$ and $k=0,1,2, \ldots$

Obviously, the sequence $\left\{E_{j}^{k}\right\}$ is a monotonically increasing sequence and the following identities are obtained.

1. $E_{j+2}^{k}=E_{j}^{k}+E_{j+1}^{k}$

2. $E_{j}^{2}=2 E_{j}^{1}$

The first 10 numbers of the sequence $\left\{E_{j}^{k}\right\}$ are represented in the table below. 


\begin{tabular}{|r|r|r|r|r|r|r|r|l|}
\hline$C$ & $C_{0}$ & $C_{1}$ & $C_{2}$ & $C_{3}$ & $C_{4}$ & $C_{5}$ & $C_{6}$ & $\ldots$ \\
\hline$j$ & $E_{j}^{0}$ & $E_{j}^{1}$ & $E_{j}^{2}$ & $E_{j}^{3}$ & $E_{j}^{4}$ & $E_{j}^{5}$ & $E_{j}^{6}$ & $\ldots$ \\
\hline 0 & 2 & 3 & 6 & 10 & 17 & 28 & 46 & $\ldots$ \\
1 & 1 & 4 & 8 & 15 & 26 & 44 & 73 & $\ldots$ \\
2 & 3 & 7 & 14 & 25 & 43 & 72 & 119 & $\ldots$ \\
3 & 4 & 11 & 22 & 40 & 69 & 116 & 192 & $\ldots$ \\
4 & 7 & 18 & 36 & 65 & 112 & 188 & 311 & $\ldots$ \\
5 & 11 & 29 & 58 & 105 & 181 & 304 & 503 & $\ldots$ \\
6 & 18 & 47 & 94 & 170 & 293 & 492 & 814 & $\ldots$ \\
7 & 29 & 76 & 152 & 275 & 474 & 796 & 1317 & $\ldots$ \\
8 & 47 & 123 & 246 & 445 & 767 & 1288 & 2131 & $\ldots$ \\
9 & 76 & 199 & 398 & 720 & 1241 & 2084 & 3448 & $\ldots$ \\
$\vdots$ & $\vdots$ & $\vdots$ & $\vdots$ & $\vdots$ & $\vdots$ & $\vdots$ & $\vdots$ & $\ddots$ \\
\hline
\end{tabular}

Table 4.1. The first 10 number of the sequence $\left\{E_{j}^{k}\right\}$

\section{Main results on the generalized Lucas sequence}

Theorem 5.1. Let $\left\{E_{j}^{k}\right\}$ be the generalized Lucas sequence, then $\lim _{j \rightarrow \infty} \frac{E_{j+1}^{k}}{E_{j}^{k}}$ converging to Golden ratio.

Proof. The proof of this theorem follows from the Theorem 3.2.

Theorem 5.2. Let $\left\{E_{j}^{k}\right\}$ be the generalized sequence of Lucas numbers, then

$$
\left(E_{j+1}^{k}\right)^{2}-E_{j}^{k} E_{j+2}^{k}=5(-1)^{j}\left[(-1)^{k}+\left(\frac{1-\sqrt{5}}{2}\right)^{k+1}+\left(\frac{1+\sqrt{5}}{2}\right)^{k+1}-1\right]
$$

is log-convex for $j=0,2,4,6, \ldots$ and log-concave for $j=1,3,5, \ldots$.

Proof. The proof of this theorem follows from the Theorem 3.2.

Theorem 5.3. For all positive integer $j$ and $k, E_{j}^{k}=\frac{5 F_{j+1} F_{k+1}+L_{j+1} L_{k+1}-2 L_{j+1}}{2}$.

Proof. The identity is proved by mathematical induction.

$\underline{\text { Case } 1 .}$ Put $k=0, j=0, E_{0}^{0}=\frac{5 F_{1} F_{1}+L_{1} L_{1}-2 L_{1}}{2}=2$.

Put $k=0, j=1, E_{1}^{0}=\frac{5 F_{2} F_{1}+L_{2} L_{1}-2 F_{2}}{2}=1$.

Hence, it holds for $k=0, j=0$ and 1 . Assume that identity is holds for fixed $k$ and $j=m$, i.e.,

$$
E_{m}^{k}=\frac{5 F_{m+1} F_{k+1}+L_{m+1} L_{k+1}-2 L_{m+1}}{2} .
$$


To prove the identity holds good for fixed $k$ and $j=m+1$, consider from Definition 4.1,

$$
E_{m+1}^{k}=L_{m+1}+L_{m+2}+L_{m+3}+\cdots+L_{m+k}+L_{m+k+1}
$$

On adding and subtracting $L_{m}$ and by using the identity (1.8) gives

$$
\begin{aligned}
E_{m+1}^{k} & =E_{m}^{k}+L_{m+k+1}-L_{m} \\
& =\frac{5 F_{m+1} F_{k+1}+L_{m+1} L_{k+1}-2 L_{m+1}}{2}+\frac{L_{m} L_{k+1}+5 F_{m} F_{k+1}}{2}-F_{m} \\
& =\frac{5 F_{m+1} F_{k+1}+L_{m+1} L_{k+1}-2 L_{m+1}+L_{m} L_{k+1}+5 F_{m} F_{k+1}-2 F_{m}}{2} .
\end{aligned}
$$

On simplification,

$$
E_{m+1}^{k}=\frac{5 F_{m+2} F_{k+1}+L_{m+2} L_{k+1}-2 L_{m+2}}{2}
$$

It holds true for a fixed $k$ and for all $j$.

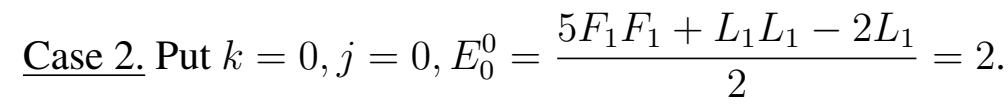

Put $k=1, j=0, E_{0}^{1}=\frac{5 F_{1} F_{2}+L_{1} L_{2}-2 L_{1}}{2}=3$.

So, it holds good for $j=0$ and $k=0$ and 1 . Assume that it holds good for a fixed $j$ and $k=m$.

$$
E_{j}^{m}=\frac{5 F_{j+1} F_{m+1}+L_{j+1} L_{m+1}-2 L_{j+1}}{2}
$$

To prove the identity holds good for a fixed $j$ and $k=m+1$, by Definition 4.1 and using the identity (1.8) gives

$$
\begin{aligned}
E_{j}^{m+1} & =E_{j}^{m}+L_{j+m+1} \\
& =\frac{5 F_{j+1} F_{m+1}+L_{j+1} L_{m+1}-2 L_{j+1}}{2}+\frac{L_{j+1} L_{m}+5 F_{j+1} F_{m}}{2} \\
& =\frac{5 F_{j+1} F_{m+1}+L_{j+1} L_{m+1}-2 L_{j+1}+L_{j+1} L_{m}+5 F_{j+1} F_{m}}{2} .
\end{aligned}
$$

On simplification,

$$
E_{j}^{m+1}=\frac{5 F_{j+1} F_{m+2}+L_{j+1} L_{m+2}-2 L_{j+1}}{2} .
$$

It holds true for a fixed $j$ for all $k$. Hence the theorem is proved. 
The result is illustrated for the first 10 values of $k$ and $j$ in the form of table below.

\begin{tabular}{|r|r|r|r|r|r|r|r|r|r|r|l|}
\hline$C$ & $C_{0}$ & $C_{1}$ & $C_{2}$ & $C_{3}$ & $C_{4}$ & $C_{5}$ & $C_{6}$ & $C_{7}$ & $C_{8}$ & $C_{9}$ & $\ldots$ \\
\hline$j$ & $E_{j}^{0}$ & $E_{j}^{1}$ & $E_{j}^{2}$ & $E_{j}^{3}$ & $E_{j}^{4}$ & $\nabla_{j}^{0}$ & $\nabla_{j}^{1}$ & $\nabla_{j}^{2}$ & $\nabla_{j}^{3}$ & $\nabla_{j}^{4}$ & $\ldots$ \\
\hline 0 & 2 & 3 & 6 & 10 & 17 & - & - & - & - & - & $\ldots$ \\
1 & 1 & 4 & 8 & 15 & 26 & -5 & -5 & -20 & -25 & -55 & $\ldots$ \\
2 & 3 & 7 & 14 & 25 & 43 & 5 & 5 & 20 & 25 & 55 & $\ldots$ \\
3 & 4 & 11 & 22 & 40 & 69 & -5 & -5 & -20 & -25 & -55 & $\ldots$ \\
4 & 7 & 18 & 36 & 65 & 112 & 5 & 5 & 20 & 25 & 55 & $\ldots$ \\
5 & 11 & 29 & 58 & 105 & 181 & -5 & -5 & -20 & -25 & -55 & $\ldots$ \\
6 & 18 & 47 & 94 & 170 & 293 & 5 & 5 & 20 & 25 & 55 & $\ldots$ \\
7 & 29 & 76 & 152 & 275 & 474 & -5 & -5 & -20 & -25 & -55 & $\ldots$ \\
8 & 47 & 123 & 246 & 445 & 767 & 5 & 5 & 20 & 25 & 55 & $\ldots$ \\
9 & 76 & 199 & 398 & 720 & 1241 & -5 & -5 & -20 & -25 & -55 & $\ldots$ \\
$\vdots$ & $\vdots$ & $\vdots$ & $\vdots$ & $\vdots$ & $\vdots$ & $\vdots$ & $\vdots$ & $\vdots$ & $\vdots$ & $\vdots$ & $\ddots$ \\
\hline
\end{tabular}

Table 5.1. The first 10 generalized Lucas numbers represent $\left(E_{j+1}^{k}\right)^{2}-E_{j}^{k} E_{j+2}^{k}$

Remark 5.1. Let $\left\{E_{j}^{k}\right\}$ be the generalized Lucas sequence, then the following identity holds.

$$
\nabla_{j}^{k}=\left(E_{j+1}^{k}\right)^{2}-E_{j}^{k} E_{j+2}^{k}=5(-1)^{j}\left[(-1)^{k}+\left(\frac{1-\sqrt{5}}{2}\right)^{k+1}+\left(\frac{1+\sqrt{5}}{2}\right)^{k+1}-1\right]
$$

is log-concave for $j=0,2,4,6, \ldots$ and log-convex for $j=1,3,5, \ldots$.

Remark 5.2. Let $\left\{E_{j}^{k}\right\}$ be the generalized Lucas sequence, then
1. $\left(E_{j+1}^{k}\right)^{2}-E_{j}^{k} E_{j+2}^{k}=-5\left[\left(D_{j+1}^{k}\right)^{2}-D_{j}^{k} D_{j+2}^{k}\right]$;
2. $E_{j+1}^{k}=E_{j}^{k+1}-L_{j}$;
3. $E_{j}^{k}-E_{j-a}^{k} E_{j+a}^{k}=5(-1)^{j+a+1}\left[(-1)^{k}+\left(\frac{1-\sqrt{5}}{2}\right)^{k+1}+\left(\frac{1+\sqrt{5}}{2}\right)^{k+1}-1\right]\left(E_{a}^{0}\right)^{2}$.

\section{Conclusion}

This article provides the generalization of Fibonacci and Lucas numbers. Few identities involving them are proved and few are stated directly. The results are illustrated for numerical values. The results studied by Zvonko Cerin [2] correspond to the columns $C_{3}, C_{7}, C_{11}, C_{15}, \ldots, C_{4 i+3}$ of Table 2.1. The results have a good number of applications in the field of medical sciences and encryption, generating OTP and develop musical nodes.

\section{Acknowledgement}

The authors grateful to expert reviewers of this article and providing valuable suggestions to improve the quality of the article. 


\section{References}

[1] Bueno, A. C. F. (2015). A note on generalized Tribonacci sequence, Notes on Number Theory and Discrete Mathematics, 21 (1), 67-69.

[2] Cerin, Z. (2013). On factors of sums of consecutive Fibonacci and Lucas numbers Annales Mathematicae et Informaticae, 41, 19-25.

[3] Chasnov, J. R. (2016). Fibonacci numbers and the Golden ratio, Lecture Notes for Coursera, The Hong Kong University of Science and Technology. Available online at: https: / / www. math.ust.hk/ machas/fibonacci.pdf.

[4] Choi, E., \& Jo, J. (2015). On partial sum of Tribonacci numbers, International Journal of Mathematics and Mathematical Sciences, 2015, Article ID 301814.

[5] Dunlap, R. A. (1997). The Golden Ratio and Fibonacci Numbers, World Scientific Press, 162 pages.

[6] Frontczak, R. (2019). Relations for generalized Fibonacci and Tribonacci sequences, Notes on Number Theory and Discrete Mathematics, 25 (1), 178-192.

[7] Irmak, N., Siar, Z., \& Keskin, R. (2019). On the sum of three arbitrary Fibonacci and Lucas numbers, Notes on Number Theory and Discrete Mathematics, 25 (4), 96-101.

[8] Khomovsky, D. I. (2018). A method for obtaining Fibonacci identities, Integers, 18, Article ID 42.

[9] Knott, R. Fibonacci Web site Available online at: http: //www . maths. surrey . ac . uk/hosted-sites/R.Knott/Fibonacci/fib.html.

[10] Komatsu, T., \& Li, R. (2019). Convolution identities for Tetranacci numbers, Notes on Number Theory and Discrete Mathematics, 25 (3), 142-169.

[11] Leyendekkers, J. V., \& Shannon, A. G. (2016). Some Golden ratio generalized Fibonacci and Lucas sequences, Notes on Number Theory and Discrete Mathematics, 22 (1), 33-41.

[12] Lokesha, V., \& Nagaraja, K. M. (2007). Relation between series and important means, Advances in Theoretical and Applied Mathematics, 2 (1), 31-36.

[13] Lokesha, V., Nagaraja, K. M., \& Kumar Naveen, B., \& Kumar, S. (2011). Solution to an open problem by Rooin, Notes on Number Theory and Discrete Mathematics, 17 (4), 33-36.

[14] Lokesha, V., Nagaraja, K. M., Kumar Naveen, B., \& Wu, Y.-D. (2011). Schur convexity of Gnan mean for two variables, Notes on Number Theory and Discrete Mathematics, $17(4), 37-41$.

[15] Melham, R. S. (1999). Families of identities involving sums of powers of the Fibonacci and Lucas numbers, Fibonacci Quarterly, 37, 315-319.

[16] Nagaraja, K. M., \& Reddy, P. S. K. (2011). Logarithmic convexity and concavity of some double sequences, Scientia Magna, 7 (2), 78-81. 